\title{
Evaluation of Cardioprotective Effects of Genistein against Diabetes-induced Cardiac Dysfunction in Rats
}

\author{
Heng-Song Tian, Guo-Qing Zhou and Zhi-Yong Zhu \\ Department of Cardiology, Pingmei ShenMa Medical Group General Hospital, Henan 467099, China
}

${ }^{*}$ For correspondence: Email: hengsong2709@gmail.com; Tel/Fax: 0086-375-2799330

Received: 6 February 2015

Revised accepted: 1 October 2015

\begin{abstract}
Purpose: To investigate the possible cardioprotective effects and potential pharmacological mechanism of genistein.

Methods: Six-week old ZDF and lean rats were randomized into 4 groups (8 rats/group), including group 1 (control lean rats); group 2 (lean rats treated with genistein, $2.5 \mathrm{mg} / \mathrm{kg}$ ); group 3 (control ZDF rats); and group 4 (ZDF treated with genistein). Two groups ( 2 and 4) were treated with genistein for 12 weeks, and cardiac functions and metabolic alterations were determined. Macrophage/monocyte chemo-attractant protein-1 (MCP-1), vascular cellular adhesion molecule-1 (VCAM-1) and intracellular adhesion molecule-1 (ICAM-1) secretion and their messenger RNA transcription level also were observed.

Results: Genistein attenuated diabetes-induced cardiac dysfunction and pathological alterations, by improving glucose tolerance and insulin resistance; facilitating Akt activation and glucose utilization, and attenuating oxidative stress and interrelated MAP kinase and NF-KB signalling pathways. In addition, genistein treatment markedly reduced diabetic-induced MCP-1 (83.33\%), VCAM-1 (74.66 \%) and ICAM-1 (71.42\%) secretion and mRNA transcription in ZDF rats.

Conclusion: The results demonstrate the putative effects of genistein against cardiovascular dysfunction by improving glucose homeostasis, attenuating oxidative stress and reduced diabeticinduced endothelial dysfunction in ZDF rats. Thus, genistein is a potential candidate for the prevention of cardiovascular diseases.
\end{abstract}

Keywords: Cardiac dysfunction, Genistein, Oxidative stress, Inflammatory response, Insulin resistance, Glucose tolerance

Tropical Journal of Pharmaceutical Research is indexed by Science Citation Index (SciSearch), Scopus, International Pharmaceutical Abstract, Chemical Abstracts, Embase, Index Copernicus, EBSCO, African Index Medicus, JournalSeek, Journal Citation Reports/Science Edition, Directory of Open Access Journals (DOAJ), African Journal Online, Bioline International, Open-J-Gate and Pharmacy Abstracts

\section{INTRODUCTION}

The American Heart Association considers diabetes to be one of the seven major controllable risk factors for cardiovascular disease. Diabetic induced cardiovascular disease was characterized in ZDF rats by increased oxidative stress, apoptosis, fibrosis, inflammation, activation of MAP kinases and NF$\mathrm{KB}$ signalling and diminished Akt phosphorylation, along with decreased glucose transport, increased cardiac lipid accumulation, and ultimately diastolic dysfunction [1,2]. The most prevalent cardiovascular complications of diabetes, characterized by both systolic and diastolic dysfunction, due to reduced contractility, prolonged relaxation and decreased compliance, are diabetic cardiomyopathy [3] and atherosclerosis [4]. Endothelial dysfunction (ED) is an early event in atherosclerosis and plays a pivotal role in the atherogenesis process [5].

The emerging experimental data suggest that cardiac insulin resistance and metabolic 
perturbations largely contribute to the development of cardiac dysfunction. The diminished glucose utilization and increased fatty acid oxidation in diabetic heart leads to lipid accumulation in myocardium [6]. An inflammatory response has already been shown to have an important role in the pathogenesis of myocardial ischemia- reperfusion injury [7]. Endothelial impairment can be detected in the early stage of atherosclerosis and serves as one of the leading mechanisms for HHcy-induced vascular dysfunction $[8,9]$.

Genistein, a major isoflavone abundant in soy, has been shown its potent vascular protective function [10,11]. Genistein could decrease oxLDL-induced ICAM-1 in human vascular endothelial cells [12], attenuate hyperglycemiainduced MCP-1 expression in human aortic endothelial cells [13], and reduce lipopolysaccharide (LPS)-induced ICAM-1 and ICAM-1 in human endothelial cells [14]. However, the mechanisms have not been clarified. In the present study, we have demonstrated the cardio protective effect of genistein and its possible mechanism involved in the protection of cardiac dysfunction.

\section{EXPERIMENTAL}

\section{Experimental animals}

Male ZDF (Zucker diabetic fatty) rats and age matched Zucker lean rats were housed in a temperature-controlled room under a 12/12 h light/dark cycle, with access to water and diet ad libitum. The 6 weeks old ZDF and lean rats were randomized into 4 groups (8 rats/group) such as group 1 -control lean rats; group 2- lean rats treated with genistein $(2.5 \mathrm{mg} / \mathrm{kg})$; group 3 control ZDF rats; group 4 - ZDF treated with genistein. Genistein was dissolved in olive oil (total concentration $1 \%$ ) and administered $1 \mathrm{~g}$ daily for 12 weeks. Body weight and fasting glucose levels were measured weekly. Before sacrificing, an oral glucose tolerance test (GTT) was performed. The animals were maintained in accordance with the Chinese legislation guidelines and the international guidelines on the protection, care and handling of laboratory animals and all the protocols were approved by the Ethical Committee of Third Affiliated Hospital of Harbin Medical University, China (approval ref. no. CLG/PSMG-GH/2014/007983).

\section{Blood chemistry and metabolic analysis}

Serum samples were collected from rats that were fasted overnight and insulin levels determined (Insulin ELISA Kit from Millipore). For the GTT, D-glucose ( $2 \mathrm{~g} / \mathrm{kg}$ body weight, Sigma Aldrich) was orally administrated after overnight fasting. Blood glucose was measured before, and after 30, 60 and 120 min of glucose uptake, using a commercially available glucometer (Bayer, IN). The area under the glucose curve (AUC) from 0-30, 0-60 and 0-120 min was calculated using the trapezoidal method [15]. Insulin sensitivity of individual animals was evaluated using the previously validated homeostasis model assessment (HOMA) index [16]. Plasma total cholesterol was measured using the polychromatic $(452,540,700 \mathrm{~nm})$ endpoint technique. The triglycerides (TG) and high-density lipoprotein (HDL) were measured using a dichromatic $(510 / 700 \mathrm{~nm} ; 600 / 700 \mathrm{~nm})$ end-point technique.

\section{Real-time RT-PCR}

The total RNA was extracted with Trizol (Invitrogen, Carlsbad, CA) according to the manufacturer's protocol. cDNA was synthesized with Revertaid First Stand cDNA Synthesis Kit (Fermentas). Real-time RT-PCR using Taq Man fluorescence methods were performed for quantitative analysis of mRNA. The products were amplified using Light Cycler II PCR machine (Roche Diagnostics, Basel, Switzerland) in the reaction mixture $(20 \mathrm{ml})$ containing $2 \mathrm{ml}$ of Light Cycler-Fast Start DNA Master Taqman (Roche, Tucson, AZ) and $0.5 \mathrm{mmol} / \mathrm{L}$ each primer. The primers specific for oxidative stress genes such as; SOD1, SOD2, Bcl2, Bax and Caspase-9; and primer specific for inflammatory marker such as; MCP-1, VCAM-1, ICAM-1, HO-1 and $\beta$-actin were designed from Integrated DNA Technologies and synthesized from invitrogen (Life Technologies). By using Light Cycler Relative Quantification Software, the amount of target gene expression was calculated as a ratio of a target transcript relative to $\beta$-actin (a housekeeping gene) in the same sample.

\section{Echocardiography}

Transthoracic echocardiography was performed in anesthetized rats using HP sonos 5500 (Hewlett-Packard) with a 12-MHz imaging transducer. Left ventricle (LV) wall thickness, diameter, systolic and diastolic function were analyzed as described previously [17], by an experienced sonographer who was blinded to treatment.

\section{Statistical analysis}

All the data were analyzed using SPSS13.0 software (SPSS Inc, Chicago, IL, USA) and each experiment was performed at least three times, 
all values were represented as mean $\pm S D ; n=8$. Comparisons between groups were tested by One-Way ANOVA analysis; $p<0.05$ was considered statistically significant

\section{RESULTS}

\section{Genistein mediated changes in body weight}

At 6 weeks of age, the ZDF rats had a higher body weight (BW) than the lean rats $(252 \pm 3 \mathrm{~g}$ vs $204 \pm 5 \mathrm{~g}$ ). The lean control rats gained BW steadily from 6 to 12 weeks, whereas the ZDF rats gained BW between 9 to 21 weeks, and there was no further gain from 21 to 25 weeks. Their BW was comparable to the lean rats at 8 weeks (333 $\pm 7 \mathrm{~g}$ in ZDF vs $350 \pm 6 \mathrm{~g}$ in the lean group) and lower at 12 weeks (340 $\pm 8 \mathrm{~g}$ in ZDF vs $320 \pm 6 \mathrm{~g}$ in the lean group, $\mathrm{p}<0.05)$. The effect of genistein on body weight was observed following 2 weeks of treatment. Rats receiving genistein for 2 weeks, significantly inhibited body weight gain in the ZDF rats. However, the untreated ZDF rats significantly gained BW through the experimental period (Table I).

\section{Genistein improve glucose homeostasis and cardiac glucose metabolism}

Fasting blood glucose levels were significantly elevated throughout the entire experimental period, in the untreated-ZDF rats (Table I), along with increased glucose tolerance (GTT), AUCGTT and HOMA-IR index at the age of 12 weeks (Fig 1, 2A), suggesting insulin resistance in ZDF rats. Genistein lowered the fasting glucose level and improved insulin resistance, as evidenced by the decreased GTT, AUCGTT and HOMA-IR index. The beneficial effect of RAR and RXR ligands on glucose metabolism was observed as early as 1 to 2 weeks following treatment (Fig 2B), Genistein significantly lowered the blood glucose level (after 2 weeks of treatment: $260 \pm$ $12 \mathrm{mg} / \mathrm{dL}$ in ZDF+Genistein vs $478 \pm 12 \mathrm{mg} / \mathrm{dL}$ in ZDF, $p<0.05$ ) and HOMA-IR index (Fig 2C) after 2 weeks of treatment. Hyperinsulinemia was observed at age 6 weeks in ZDF rats $(6.08 \pm$ $0.75 \mathrm{ng} / \mathrm{dL}$ in ZDF vs $0.44 \pm 0.03 \mathrm{ng} / \mathrm{dL}$ in lean, $p$ $<0.05)$. At age 12 weeks, the serum insulin level in the ZDF rats was decreased to a level comparable with the lean rats $(0.71 \pm 0.07 \mathrm{ng} / \mathrm{dL}$ in ZDF vs $0.76 \pm 0.09 \mathrm{ng} / \mathrm{dL}$ in lean, (Fig. 2). This observation was accompanied by a significantly increased HOMA-IR index and decreased HOMA- $\beta \%$ index, suggesting that impaired $\beta$ cell function may contribute to the decreased insulin level and progression of the diabetic process. At the age of 12 weeks, serum insulin was maintained at a higher level in the genistein treated ZDF rats $(p<0.05$, vs ZDF), along with a decreased HOMAIR index and increased HOMA$\beta \%$ index, (Fig. 2D), suggesting that genistein treatment has beneficial effects on improving insulin resistance and $\beta$ cell function.

\section{Genistein inhibit oxidative stress, apoptosis and activation of MAP kinases in ZDF rat hearts}

The results showed a significantly increased intracellular ROS generation (Fig. 3) and decreased expression of SOD1 and SOD2 in Figure 3A were observed in the ZDF rat's hearts. Increased apoptosis was also observed in the ZDF rat's hearts,

Table 1: Basic parameters for the study

\begin{tabular}{|c|c|c|c|c|}
\hline & Lean & Lean+genistein & ZDF & ZDF+ genistein \\
\hline \multicolumn{5}{|l|}{ Body weight (g) } \\
\hline 0 week & $204 \pm 5$ & $199 \pm 2$ & $252 \pm 3$ & $251 \pm 5$ \\
\hline 4 weeks & $258 \pm 6$ & $280 \pm 4$ & $317 \pm 7$ & $288 \pm 9$ \\
\hline 8 weeks & $313 \pm 4$ & $307 \pm 4$ & $333 \pm 7$ & $302 \pm 17^{\#}$ \\
\hline 12 weeks & $320 \pm 6$ & $293 \pm 4$ & $340 \pm 8$ & $292 \pm 23^{*}$ \\
\hline \multicolumn{5}{|l|}{ Blood glucose (mg/dL) } \\
\hline 0 week & $63 \pm 2$ & $86 \pm 29$ & $73 \pm 5$ & $94 \pm 5$ \\
\hline 4 weeks & $77 \pm 3$ & $74 \pm 7$ & $324 \pm 18$ & $161 \pm 12$ \\
\hline 8 weeks & $79 \pm 42$ & $76 \pm 4$ & $336 \pm 12$ & $188 \pm 17$ \\
\hline 12 weeks & $75 \pm 4$ & $71 \pm 4$ & $438 \pm 25^{x}$ & $161 \pm 34^{\pi}$ \\
\hline \multicolumn{5}{|l|}{ At the end of the study } \\
\hline $\mathrm{HR}(\mathrm{bpm} / \mathrm{min})$ & $264 \pm 5$ & $261 \pm 9$ & $232 \pm 7$ & $247 \pm 12$ \\
\hline $\mathrm{HW}(\mathrm{g})$ & $0.90 \pm 0.04$ & $0.81 \pm 0.04$ & $1.15 \pm 0.03$ & $0.85 \pm 0.02^{\#}$ \\
\hline HW/BW (mg/g) & $1.70 \pm 0.06$ & $1.79 \pm 0.05$ & $2.95 \pm 0.03$ & $2.42 \pm 0.08$ \\
\hline $\mathrm{HW} / \mathrm{TL}(\mathrm{mg} / \mathrm{mm})$ & $17.3 \pm 1.04$ & $15.8 \pm 0.31$ & $23.4 \pm 0.86$ & $19.0 \pm 0.49^{\#}$ \\
\hline
\end{tabular}

Treatment started at the age of 6 weeks, 0 week = before treatment; HR = heart rate; $H W=$ heart weight; $B W$ - body weight; $T L=$ tibia length; ${ }^{*} p<0.05$, vs lean; $\# p<0.05$, vs ZDF 


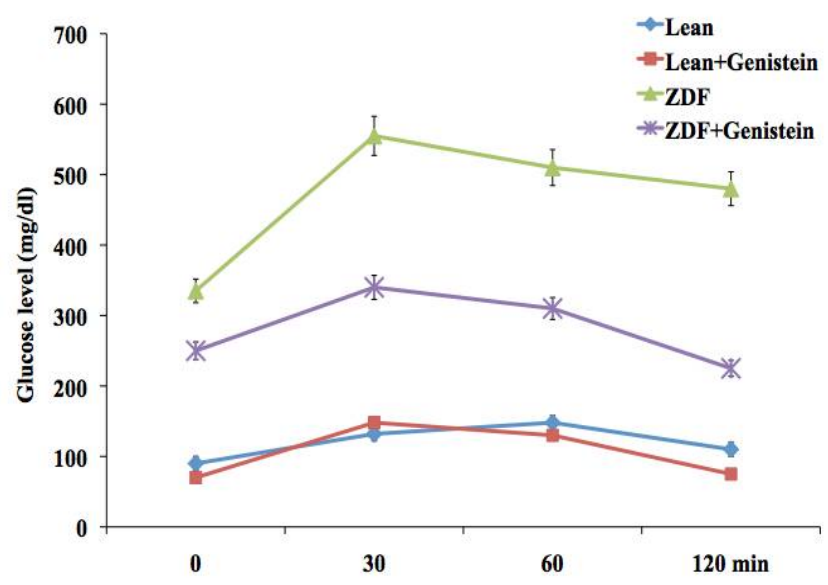

Figure 1: Represent the glucose level monitored during GTT in lean and ZDF rats with the treatment of genistein. Glucose levels were measured at $0,30,60$ and $120 \mathrm{~min}$ following glucose uptake. Values are expressed as mean \pm SEM $(n=8) ;{ }^{*} p<0.05$ vs ZDF group

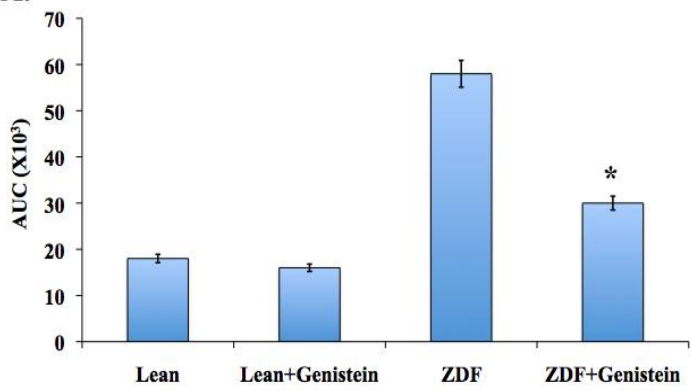

C.

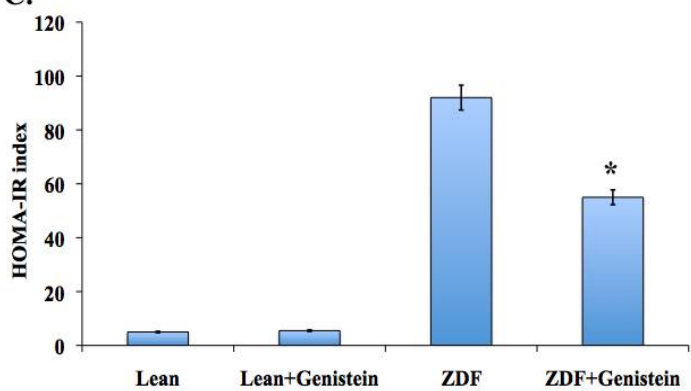

B.

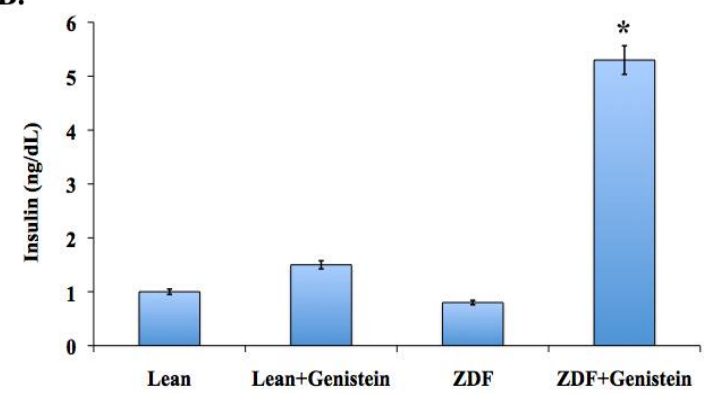

D.

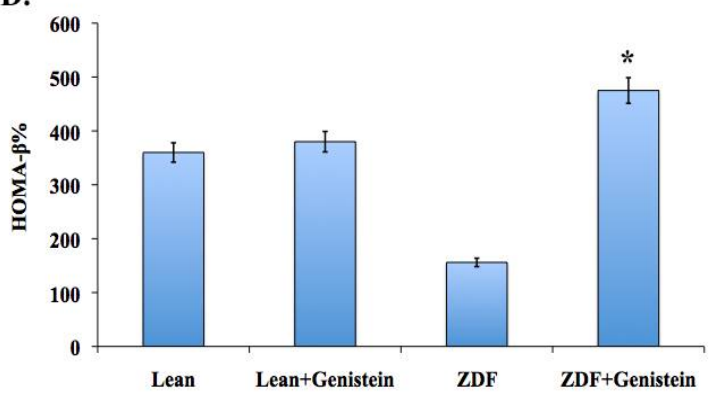

Figure 2: Represent the measurement of AUC based on GTT results (A). The level of plasma insulin (B), HOMA-IR (C) and HOMA- $\beta \%$ (D) were determined at the end of the experiment. Values are expressed as the mean \pm SEM $(n=8) .{ }^{*} p<0.05$ vs ZDF group

as evidenced by decreased expression of Bcl2, a decreased $\mathrm{Bcl} 2 / \mathrm{Bax}$ ratio and increased expression of Bax, caspase 9 and TUNELpositive myocytes (23\%) (Fig. 3B, C). Genistein inhibited ROS generation and the activated MAP kinases and apoptotic signaling, as well as the percent of TUNEL-positive myocytes (10 and 2.3 $\%$, respectively, $p<0.05$, vs ZDF). These data suggest that genistein inhibits apoptosis of cardiomyocyte, by reducing oxidative stress and associated MAP kinase pathways.

\section{Effects of genistein on mRNA levels of MCP- 1, VCAM-1, ICAM-1 and HO-1}

The mRNA levels of MCP-1, VCAM-1 and ICAM1 were analyzed using real-time RT-PCR. The mRNA levels in untreated ZDF rats showed a marked increases of MCP-1 (9.09\%), VCAM-1 $(20 \%)$ and ICAM-1(21.42 \%) secretion compared with lean $(p<0.05)$; however, treatment with genistein would significantly inhibit MCP-1 (83.33\%), VCAM-1 (74.66 \%) and ICAM$1(71.42 \%)$ secretion compared with ZDF rats (Fig 4A).

Trop J Pharm Res, November 2015; 14(11): 2018 
A.

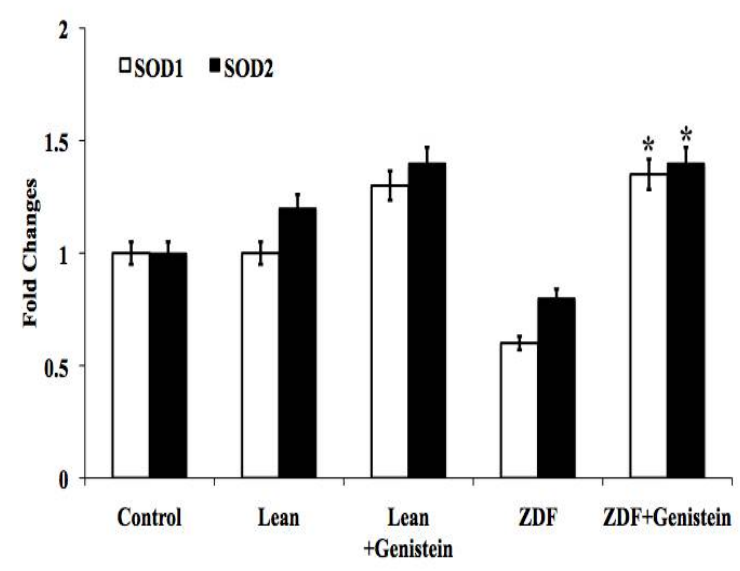

B.

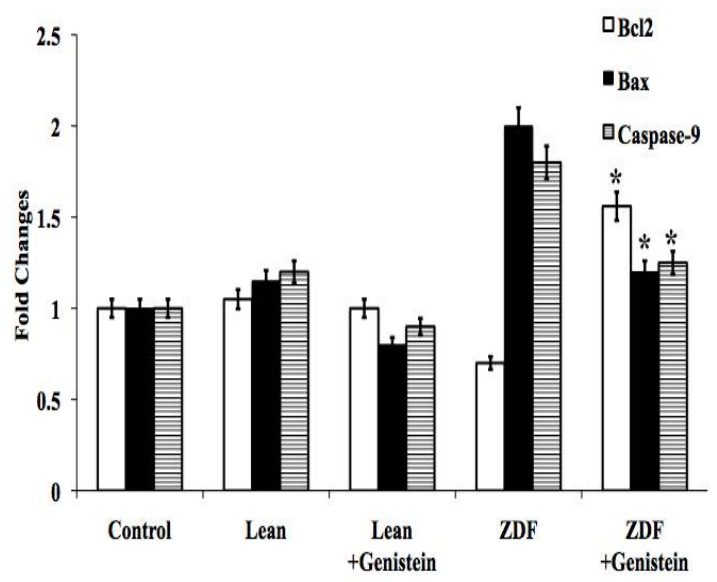

C.

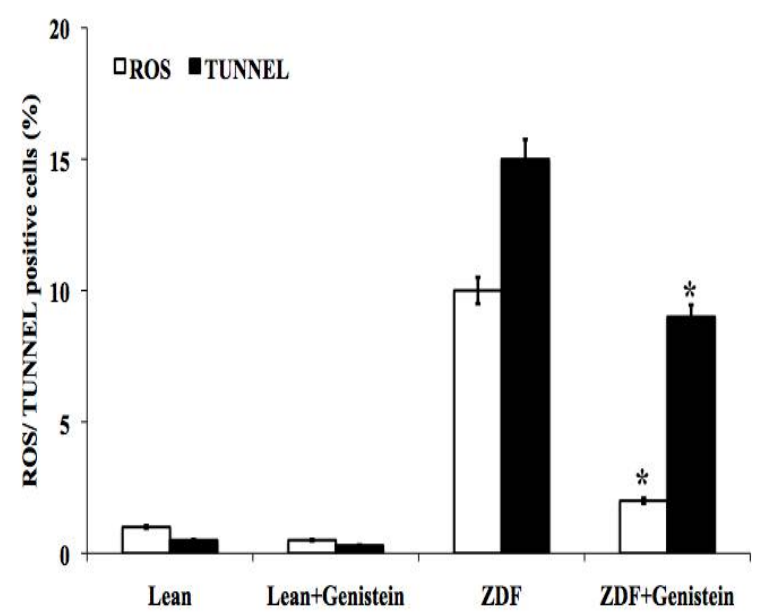

Figure 3: Represent the effect of genistein against oxidative stress in Lean and ZDF rats. The mRNA levels of SOD1 \& SOD2 (A), the mRNA levels of Bcl2, Bax and Caspase 9 (B), and the graphical representation of percentage of ROS stainging \& TUNNEL positive cells (C). Values are expressed as the mean \pm SEM $(n=3) ;{ }^{*} p<0.05$ vs ZDF group
This result suggests the inhibitory effects of genistein on pro-inflammatory cytokine secretion. The mRNA level of HO-1 was significantly $(p<$ 0.05 ) increased by genistein administration in both the lean and ZDF rats (Fig. 4B). Interestingly, our report demonstrate that genistein control the inflammatory marker by enhancing the $\mathrm{HO}-1$ expression.

A.

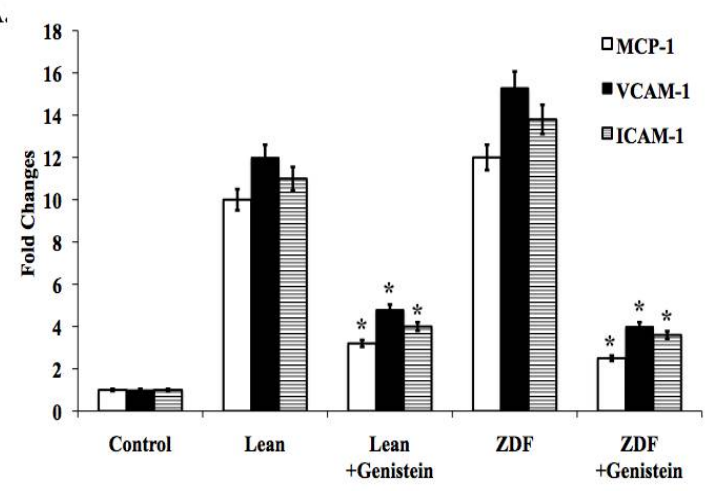

B.

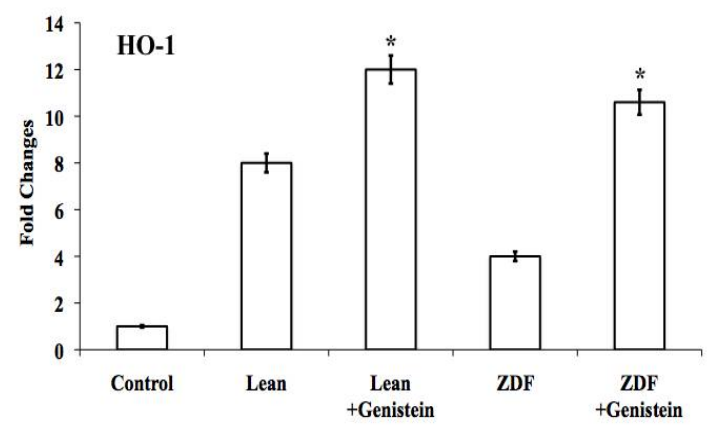

Figure 4: Represent the mRNA levels of inflammatory markers $(A)$ and $\mathrm{HO}-1$ (B) of cardiac tissue from the experimental groups. Values are expressed as mean \pm SEM $(\mathrm{n}=8)$. ${ }^{*} p<0.05$, vs ZDF group

\section{Effect of genistein on left ventricular structural and functional changes}

There was no difference observed in heart rate between the groups (Table I). Increased heart weight $(\mathrm{HW}), \mathrm{HW} / \mathrm{BW}$ ratio and HW/TL (tibia length) ratio were observed in untreated-ZDF rats $(p<0.05$, vs lean control). Due to changes in the body weight between groups, we used the $\mathrm{HW} / \mathrm{TL}$ ratio, as an index, to compare the effect of genistein on cardiac hypertrophy. Genistein significantly inhibited the increased $\mathrm{HW} / \mathrm{TL}$ in ZDF rats $(29 \pm 0.49 \mathrm{mg} / \mathrm{mm}$ in ZDF + Am vs 33.4 $\pm 0.86 \mathrm{mg} / \mathrm{mm}$ in ZDF, $p<0.05)$. A significantly increased thickness of the systolic posterior wall of the LV (LVPWs), along with decreased systolic inner diameter of the LV was observed in untreated ZDF rats at 12 weeks of age (Fig 5A), representing a concentric cardiac hypertrophy, which was not observed in genistein treated ZDF 
rats. The increased HW/TL and LVPWs were in line with the increased expression of the hypertrophic markers ANP and BNP (Fig 5B). There was no significant decrease in LV ejection fraction (LVEF) and fractional shortening (FS) in ZDF rats, suggesting no significant systolic dysfunction in ZDF rats, at 12 weeks of age. However, a significantly decreased E/A ratio, and increased IVRT and DT were observed (data not shown) in ZDF rats, at 8 and 12 weeks of age ( $p$ $<0.05$, vs lean rats), indicating that diastolic function was impaired.

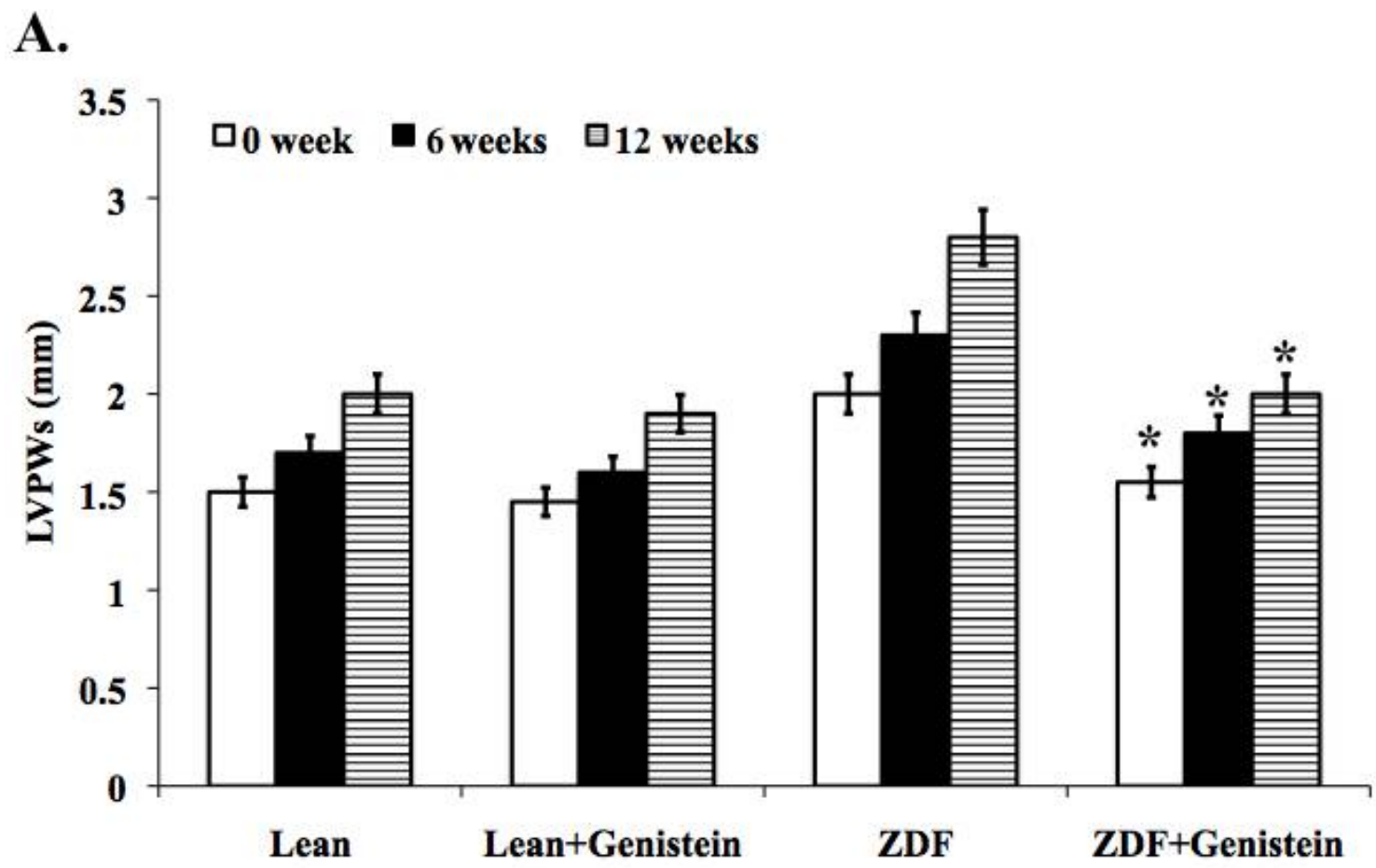

B.

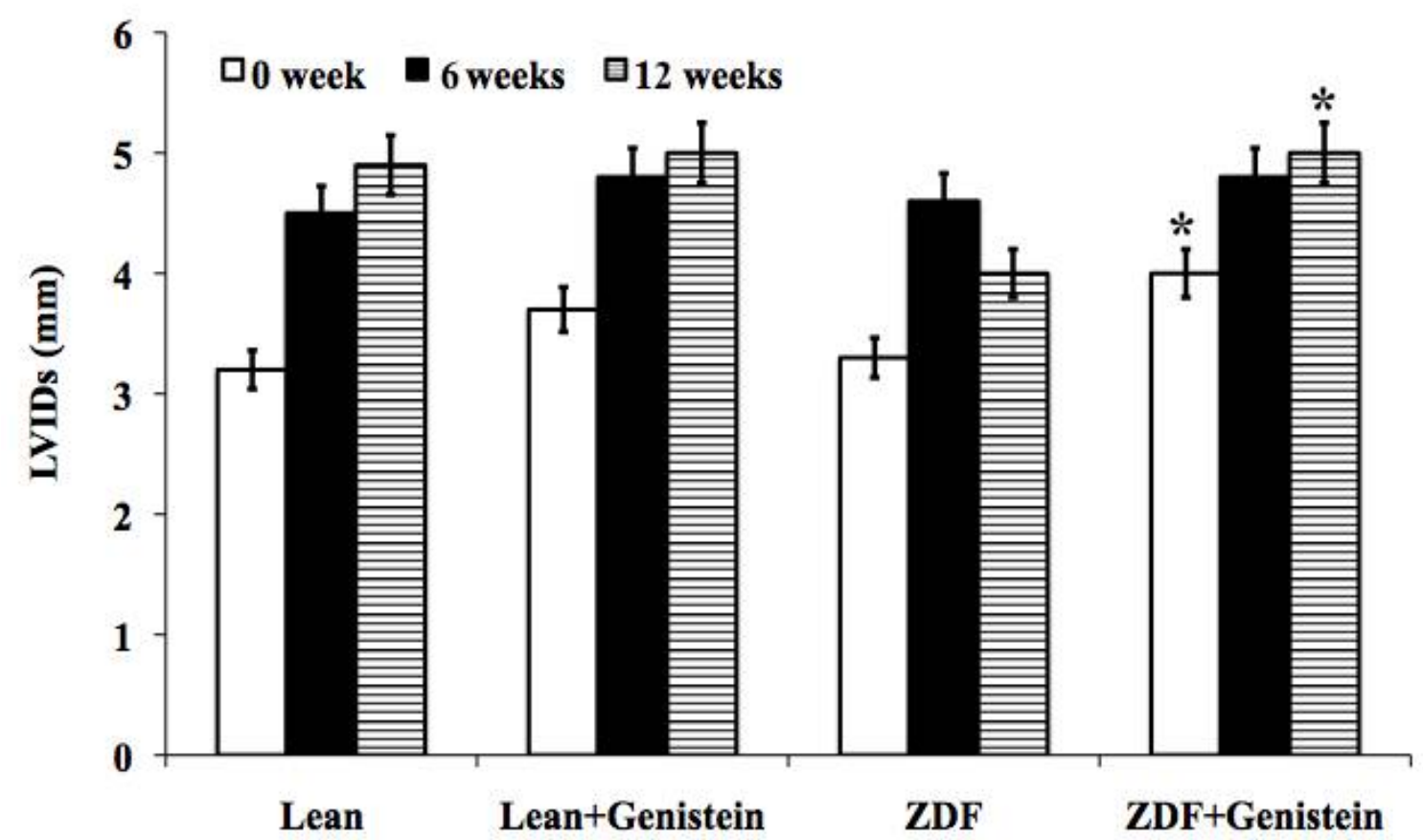

Figure 5: Represent the Echocardiographic studies performed for 6 and 12 weeks in lean and ZDF rats administered with genistein with a special focus to LVPWs (A) and LVIDs (B). Values are expressed as the mean $\pm \operatorname{SEM}(\mathrm{n}=8) ;{ }^{*} p<0.05$, vs ZDF group 


\section{DISCUSSION}

The present study demonstrated that genistein ameliorated diabetes-induced cardiac dysfunction and other pathological alterations. Genistein, a major isoflavone abundant in soy, has been shown its potent cardiovascular protective function [10,11]. The beneficial effects of genistein may be associated with the mechanisms like improvement of the impaired cardiac insulin metabolic signalling by reducing systemic hyperglycemia and insulin resistance; enhancement of cardiac glucose uptake/utilization and reducing glucotoxityinduced oxidative stress; inhibition of MAP kinases activation and amelioration of cardiac inflammatory responses.

In the present study, hyperinsulinemia, hyperglycemia, hyperlipidemia and insulin resistance were observed in ZDF rats. Diabetic induced cardiac dysfunction as evidenced by diastolic dysfunction with hypertrophy, fibrosis, inflammation and apoptosis developed in ZDF rats.

Our results showed significantly increased HOMA-IR index and decreased HOMA- $\beta \%$ index in ZDF rats, suggesting that impaired $\beta$ cell function may contribute to the decreased insulin level and development of the diabetes. This observation was consistent with previous studies, which showed a decreased serum insulin level in aged ZDF rats $[18,19]$. A number of factors could contribute to the pathological effects observed. The hyperglycemia and hyperlipidemia-induced intracellular generation of ROS can act as signal transduction molecules to activate various signalling pathways, which ultimately lead to an inflammation, a cell apoptosis and fibrosis $[20,21]$. We observed significantly increased oxidative stress, apoptosis, inflammatory responses and activation of MAP kinases in ZDF rats. However, genistein administration have prevented these effects may be due to the antioxidation and anti-angiogenesis effects as well as estrogenic activities of genistein [22]. Increased ROS (reactive oxygen species) formation activates MAP kinase-signalling pathways and promotes cardiomyocyte apoptosis [23,24]. Our data further confirmed that diabetes-induced oxidative stress plays vital role in regulation of the development of diabetic complications. Insulin resistance reduces phosphorylation of Akt pathway, which is involved in regulation of genes that control glucose and lipid homeostasis and other cellular processes like cellular hypertrophy, protein translation, nitric oxide generation, and apoptosis $[25,26]$. The mechanisms involved in cardio protective function of genistein are obscure. Besides its non-specific tyrosine kinase inhibitory role, genistein could exert vascular protective action through many other signaling pathways, such as cAMP/protein kinase $A$ and PI3 K/AKT pathways [27].

Furthermore, we analyzed the cardiac inflammatory response, where the increased mRNA level of MCP-1, VCAM-1 and ICAM-1 found in untreated ZDF rats. However, genistein significantly inhibited diabetic-induced MCP-1, VCAM-1 and ICAM-1 expression. This is consistent with the previous report that genistein increased heme oxygenase (HO) activity, where $\mathrm{HO}$ system is tightly involved in the inhibitory activities of genistein against inflammation [28]. This part of our study showed that the down regulation of the expression levels of MCP-1, VCAM-1 and ICAM-1 was achieved by genistein mediated induction of $\mathrm{HO}-1$.

\section{CONCLUSION}

Altogether, the findings reveal the protective effect of genistein on cardiovascular dysfunction by maintaining glucose homeostasis and cardiac glucose metabolism, inhibiting oxidative stress, apoptosis and activation of MAP kinases and prevention of inflammatory response. Thus, administration of genistein against cardiovascular diseases may be a positive approach to the development of a potential drug.

\section{REFERENCES}

1. Daniels $A$, Linz $D$, van Bilsen $M$, Rutten $H$, Sadowski $T$, Ruf $S$, Juretschke HP, Neumann-Haefelin $C$, Munts $C$, vander Vusse GJ, van Nieuwenhoven FA. . Longterm severe diabetes only leads to mild cardiac diastolic dysfunction in Zucker diabetic fatty rats. Eur $J$ Heart Fail 2012; 14: 193-201.

2. Baynes J, Murray DB. Cardiac and renal functions are progressively impaired with aging in Zucker diabetic fatty type II diabetic rats. Oxid Med Cell Longev 2009; 2: 328-334.

3. Murarka S, Movahed MR. Diabetic cardiomyopathy. J Card Fail 2010; 16: 971-979.

4. Werner N, Nickenig G. Influence of cardiovascular risk factors on endothelial progenitor cells: limitations for therapy? Arterioscler Thromb Vasc Biol 2006; 26: 257-266.

5. Bonetti PO, Lerman LO, Lerman A. Endothelial dysfunction: a marker of atherosclerotic risk. Arterioscler Thromb Vasc Biol 2003; 23: 168-175.

6. Feuvray D, Darmellah $A$. Diabetes-related metabolic perturbations in cardiac myocyte. Diabetes Metab 2008; 34: S3-S9. 
7. Lucchesi, BR. Modulation of leukocyte-mediated myocardial reperfusion injury. Annu Rev Physiol 1990; 52: 561- 566.

8. Kanani PM, Sinkey CA, Browning RL, Allaman M, Knapp $H R$, and Haynes WG. "Role of oxidant stress in endothelial dysfunction produced by experimental hyperhomocyst(e)inemia in humans," Circulation 1999; 100: 1161-1168.

9. Eberhardt RT, Forgione MA. Cap A, Leopold JA, Rudd $M A$, Trolliet $M$, Heydrick $S$, Stark $R$, Klings $E S$, Moldovan NI et al. "Endothelial dysfunction in a murine model of mild hyperhomocyst(e)inemia," J Clin Investig 2000; 106: 483-491.

10. Si $\mathrm{H}$, and Liu D. "Phytochemical genistein in the regulation of vascular function: new insights," Curr Med Chem 2007; 14: 2581-2589,

11. Rimbach G, Boesch-Saadatmandi C, Frank J. Fuchs $D$, Wenzel U, Daniel H, Hall WL, Weinberg PD. "Dietary isoflavones in the prevention of cardiovascular disease-a molecular perspective," Food Chem Toxicol, 2008; 46: 1308-1319.

12. Yi L, Jin $X$, Chen $C Y$, Fu YJ, Zhang T, Chang H, Zhou $Y$, Zhu JD, Zhang $Q Y$, and Mi MT. * Chemical structures of 4-oxo-flavonoids in relation to inhibition of oxidized low-density lipoprotein (LDL)-induced vascular endothelial dysfunction. Int J Mol Sci 2011; 12: 5471 5489.

13. Babu PV, Si H, Fu Z, Zhen W, Liu D. *Genistein prevents hyperglycemia-induced monocyte adhesion to human aortic endothelial cells through preservation of the cAMP signaling pathway and ameliorates vascular inflammation in obese diabetic mice. J Nutr 2012; 142: 724-730.

14. De Andrade CM, De Sa MF, Toloi MR. Effects of phytoestrogens derived from soy bean on expression of adhesion molecules on HUVEC. Climacteric 2012; 15: $186-194$.

15. Purves RD. Optimum numerical integration methods for estimation of area under-the-curve (AUC) and areaunder-the-moment-curve (AUMC). J Pharmacokinet Biopharm 1992; 20: 211-226.

16. Matthews DR, Hosker JP, Rudenski AS, Naylor BA, Treacher DF, Turner RC. Homeostasis model assessment: insulin resistance and beta-cell function from fasting plasma glucose and insulin concentrations in man. Diabetologia 1985; 28: 412419.

17. Choudhary R, Palm-Leis A, Scott RC, Guleria RS, Rachut E, Baker KM. All-trans retinoic acid prevents development of cardiac remodeling in aortic banded rats by inhibiting the renin-angiotensin system. Am J Physiol Heart Circ Physiol 2008; 294: H633-H644.

18. Fulop N, Mason MM, Dutta K, Wang P, Davidoff $A J$, Marchase RB. Impact of Type 2 diabetes and aging on cardiomyocyte function and O-linked $\mathrm{N}$ acetylglucosamine levels in the heart. Am J Physiol Cell Physiol 2007; 292: C1370-C1378.

19. Toblli J, Cao G, Rivas C, Munoz M, Giani J, Dominici F. Cardiovascular protective effects of nebivolol in Zucker diabetic fatty rats. J Hypertens 2010; 28:1007-1019.

20. Selvaraju V, Joshi M, Suresh S, Sanchez JA, Maulik N, Maulik G. Diabetes, oxidative stress, molecular mechanism, and cardiovascular disease--an overview. Toxicol Mech Methods 2012; 22: 330-335.

21. Khullar M, Al-Shudiefat AA, Ludke A, Binepal G, Singal PK. Oxidative stress: a key contributor to diabetic cardiomyopathy. Can J Physiol Pharmacol 2010; 88: 233-240.

22. Su SJ, Yeh TM, Chuang WJ, Ho CL, Chang KL, Liu HS, Cheng HL, Hsu PY, Chow NH. The novel targets for anti-angiogenesis of genistein on human cancer cells. Biochem Pharmacol 2005; 69: 307-18.

23. Thandavarayan RA, Watanabe K, Ma M, Gurusamy N, Veeraveedu PT, Konishi T. Zhang S, Muslin AJ, Kodama M, Aizawa Y. Dominant-negative p38alpha mitogen-activated protein kinase prevents cardiac apoptosis and remodeling after streptozotocininduced diabetes mellitus. Am J Physiol Heart Circ Physiol 2009; 297: H911-H919.

24. Tsai KH, Wang WJ, Lin CW, Pai P, Lai TY, Tsai CY, Kuo WW. NADPH oxidase-derived superoxide anioninduced apoptosis is mediated via the JNKdependent activation of NF-kappaB in cardiomyocytes exposed to high glucose. J Cell Physiol 2012; 227: 1347-1357.

25. Muniyappa R, Montagnani M, Koh KK, Quon MJ. Cardiovascular actions of insulin. Endocr Rev 2007; 28: 463-491.

26. Rask-Madsen C, Kahn CR. Tissue-specific insulin signaling, metabolic syndrome, and cardiovascular disease. Arterioscler Thromb Vasc Biol 2012; 32: 2052-2059.

27. Zheng FL, Zhao JH, Zhang HP, "The action of PI3K/AKT during genistein promoting the activity of eNOS," Zhonghua Xin Xue Guan Bing Za Zhi, 2012; 40: 327331.

28. Zhang HP, Zheng FL, Zhao JH, Guo DX, Chen XL. Genistein inhibits ox-LDL-induced VCAM-1, ICAM-1 and MCP-1 expression of HUVECs through heme oxygenase-1. Arch Med Res 2013; 44:13-20. 